\title{
Impact of awareness in metapopulation epidemic model to suppress the infected individuals for different graphs
}

\author{
K.M. Ariful Kabir ${ }^{1,2, a}$ and Jun Tanimotoc ${ }^{3}$ \\ ${ }^{1}$ Interdisciplinary Graduate School of Engineering Sciences, Kyushu University, Kasuga-koen, Kasuga-shi, Fukuoka \\ 816-8580, Japan \\ ${ }^{2}$ Department of Mathematics, Bangladesh University of Engineering and Technology, Dhaka, Bangladesh \\ ${ }^{3}$ Faculty of Engineering Sciences, Kyushu University, Kasuga-koen, Kasuga-shi, Fukuoka 816-8580, Japan
}

Received 26 September 2018 / Received in final form 29 June 2019

Published online 9 September 2019

(C) EDP Sciences / Società Italiana di Fisica / Springer-Verlag GmbH Germany, part of Springer Nature, 2019

\begin{abstract}
The metapopulation dynamical model with information spreading in SIS epidemic diffusion model is presented for random walkers for sub-populations to display the effect of awareness. Two-layer SIS-UA (susceptible-infected-susceptible-unaware-aware) epidemic model is considered to reveal the effect of information spreading in a graph, where, each node denoted a sub-population. The individuals migrate by random walk from one node to another node on the graph by themselves or forcefully to escape from contagious disease. Moreover, the individuals in a node are classified into four states as unaware susceptible (US), aware susceptible (AS), unaware infected (UI) and aware infected (AI). Meanwhile, to study the impact of graph topology on individuals in each node (subpopulation), four different graphs: star, cycle, wheel and complete are considered as representing both homogeneous and heterogeneous connections with the various number of nodes. The influence of migration for information spreading is displayed to subdue infected individuals with time steps. Finally, several impressive cases in terms of what attributes of individuals in each subpopulation being allowed (or say, pushed) migration are considered, which is summarized in the form of a full phase diagram.
\end{abstract}

\section{Introduction}

Metapopulation dynamics in ecology implies the migration of unstable local populations even for human activities; such as natural disasters and contagious diseases that make the population into patches. The epidemic SIS model [1], where S and I meaning a susceptible and infected individual can be applied to consider showing the impact of migration from different groups of the population. In metapopulation, each local habitat is said subpopulation of a species, where, generally speaking, individual moves from a higher to a lower or a lower to a higher density patch. Moreover, the habitat patch area, migration, isolation, colonization, and extinction are integrated as the result of classic metapopulation dynamics, that can be explained the pattern of individual's movement, distribution, choice and the dynamics of the population in real fragmented landscapes [2]. The present study focuses on how individual migration affecting disease and information spreading in a society, which may help to give qualitative and quantitative knowledge to the authority for the prevention of epidemic.

In the current work, we consider random migration, that is to say, each individual can select their destination

\footnotetext{
${ }^{a}$ e-mail: k.ariful@yahoo.com
}

randomly [3]. Random walk bringing migration is a very fundamental mechanism to many diseases spreading processes on graphs that have been studied by many researchers [4-11]. In epidemic diffusion on a network, every subpopulation is considered as a node and a link between nodes is called path or edge, whereas, disease and information spreading are occurred concurrently in a node (subpopulation) [12]. Recently, Nagatani et al. [13] investigated the metapopulation model for random walkers for the star, cycle and complete graphs to show where the epidemic is identical and the infection rate is unchanged for any nodes. We mainly concentrate on four different types of graphs; star, cycle, wheel and complete to introduce the impact of information in epidemic SIS dynamic for $N$ nodes in subpopulation migration model.

Incidentally, the effect of awareness with the diffusion of diseases has a great impact to lessen infection and alert people to take self-protection or quarantine in a safe place. Moreover, people perform not only as a medium of disease spreader but also information propagator in the context of the dynamical system. A two-layer compartment epidemic model SIS (susceptible-infected-susceptible) is considered for unaware and aware (UA) situation to elucidate the physical and virtual layer in a complex population network [14-16]. To understand the mechanics of such a complex dynamical system and draw some appropriate results, 
rather than a theoretical method, the MAS approach is suitable. In the framework of SIR/V model, Kuga et al. [17] explored an analytical epidemic vaccination game theory model with MAS approach that described the impact of an imperfect vaccination and an intermediate defense measure. Meanwhile, the epidemic spreading model has a rich research history including susceptibleinfected (SI) model [18], susceptible-infected-susceptible (SIS) model [19] and susceptible-infected-recovered (SIR) model [20]. Furthermore, information spreading "awareness" with disease spreading will help people to take intermediate protection like wearing a mask, washing hand and mosquito net to oppress the infection diffusion by force or self-interest. Many epidemics with information model studied to display the impact of information on the social complex network [21-27]. Fukuda et al. [28] explored two-layer network for infection spreading and information spreading called physical network and virtual network by taking a MAS approach, although 'information spreading' in her model does not mean the dissemination of somehow useful information for disease protection but indicates individuals' strategy updating; either committing vaccination or not. Recently, Moinet et al. [29] investigated the SIR model with awareness effect on Active-Driven temporal networks about the disease transmittance decaying with the increase of infected neighbors around a focal individual for his awareness. Following to reference [13], our model deals with information spreading, in which awareness can help individuals to migrate from the worst place to safer one to lessen the contagious diseases and also present some assumption that can help the authority to take action to reduce the disease in an area.

In this paper, the rest of the work is prepared as follows: coupled SIS-UA epidemic for metapopulation model is explored for four different graphs: star, cycle, wheel and complete with schematic diagram in Section 2. In Section 3, the numerical simulation with result and discussion is done to investigate the impact of awareness by drawing the 2D full phase diagrams. Finally, in Section 4, the conclusion of the paper are presented.

\section{SIS-UA metapopulation model}

The SIS-UA coupled epidemic dynamics with information spreading is explored by using the compartment model for the metapopulation. In this model, the metapopulation is considered into $N$ subpopulations which called nodes, where individuals migrate freely from subpopulation by a simple random walk. The link among subpopulation is presented by graph and the individuals randomly walk among nodes through links by considering four graphs: star, cycle, wheel and complete. The individuals in each subpopulation can be classified into unaware susceptible $(U S)$, aware susceptible $(A S)$, unaware infected $(U I)$ and aware infected $(A I)$. The mean field dynamics is assumed to represent inside each subpopulation for SIS with two-layer unaware and aware situations and the connection between those two layers visually explained in Figure 1.
From Figure 1, it can be explicated that, the SIS-UA metapopulation depicts the effect of awareness for a random walk from different subpopulations. In a subpopulation, the individuals of unaware susceptible may become infected at the disease transmission rate $\beta$. The unaware susceptible can be aware of susceptible at the information transmission rate $\alpha$. But, the aware susceptible individuals can reduce risk of infection by using intermediate defense measure and may also become infectious at the rate of $(1-\eta) \beta$, where, $\eta ; 0 \leq \eta \leq 1$, is the rate of self-protection from aware susceptible to aware infected. Both unaware and aware infected individuals become susceptible individuals at the rate $\gamma$. Meanwhile, the connection between unaware susceptible to aware susceptible is described as virtual interaction for the information spreading and the disease spreading is defined as physical interaction. In order to formulate the SIS-UA epidemic model for metapopulation migration model, the mean field approach with two-layer compartment model is applied. The population is divided into $N$ subpopulations of which population density is denoted as $\rho_{1}, \rho_{2}, \rho_{3}, \ldots, \rho_{N}$. The individuals are moving from one subpopulation to another at the same migration rate $m$. The individuals of each node are classified into four states as unaware susceptible $U S_{k}$, aware susceptible $A S_{k}$, unaware infected $U I_{k}$ and aware infected $A I_{k}$. As constraint, we presume; $\rho_{k}(t)=U S_{k}(t)+$ $A S_{k}(t)+U I_{k}(t)+A I_{k}(t)$ for local and $\rho_{\text {total }}(t)=$ $\sum_{k \in N}\left(U S_{k}(t)+A S_{k}(t)+U I_{k}(t)+A I_{k}(t)\right)=1 \quad$ for global, where, $k=1,2,3, \ldots N$ and $N$ is the number of subpopulations (nodes). In the first case, we consider only aware infected individuals can migrate from each subpopulation to other according to what the pioneer study presumed [13]. With these assumptions, the mean field approach is given by the set of the following system of ordinary differential equations for four different graphs: star, cycle, wheel and complete for $N$ subpopulation.

\subsection{Star graph}

A graph with one internal node (hub node) and $N-1$ leaves connected with hub node is called star graph for $N$ nodes in Figure 2a (as for example $N=7$ ). In star graph of $N$ vertices, the number of connection or edges are $N-1$. Every nodes other than hub node have only one connection and the hub node has $N-1$ links. The SIS-UA epidemic for metapopulation model is as follows, where we define the migration rate obeying to random walk as $m$.

\section{Subpopulation model}

$$
\begin{aligned}
\frac{d \rho_{1}(t)}{d t} & =\sum_{i \in N, i>1} m \rho_{i}(t)-m \rho_{1}(t) \\
k & =1 \text { (focal point; central node) } \\
\frac{d \rho_{k}(t)}{d t} & =\frac{1}{N-1} m \rho_{1}(t)-m \rho_{k}(t) \\
k & >1, k=2,3,4, \ldots N
\end{aligned}
$$




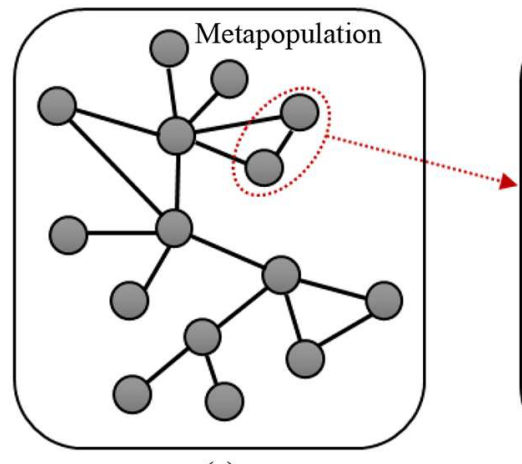

(a)

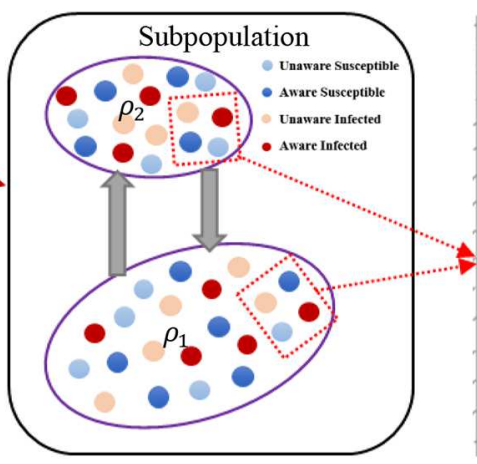

(b)

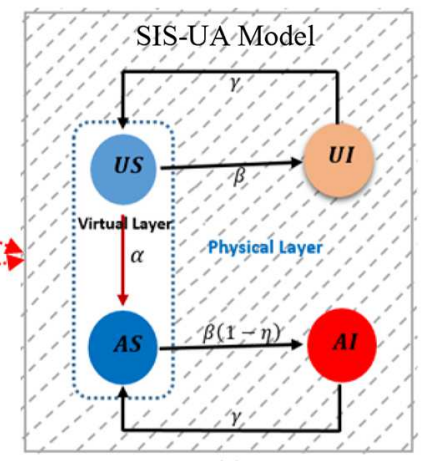

(c)

Fig. 1. Schematic diagram of (a) the metapopulation model, (b) the subpopulation with four types of individuals, (c) the two layer epidemic SIS-UA model.

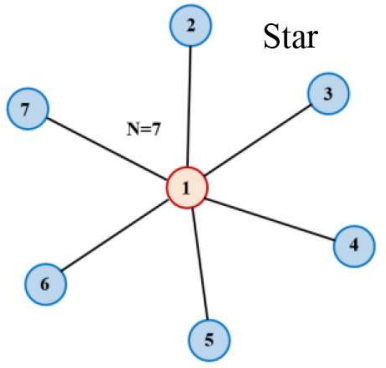

(a)

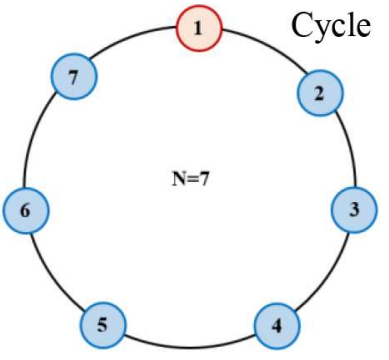

(b)

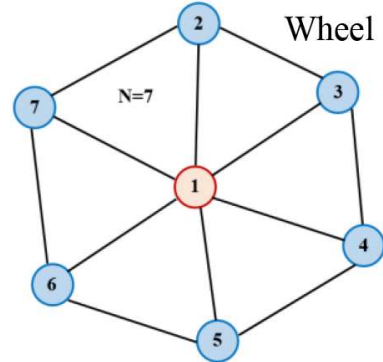

(c)

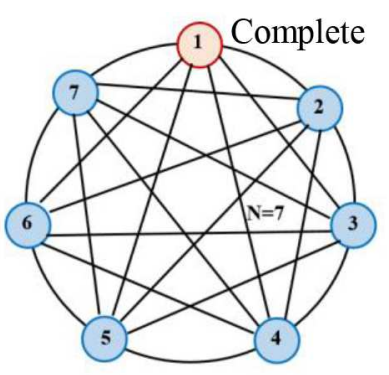

(d)

Fig. 2. The metapopulation model of seven nodes $(N=7)$ for four graphs: (a) star, (b) cycle, (c) wheel and (d) complete. Node 1 is considered as a hub nodes for all graphs.

\section{SIS-UA model}

For $k=1$ (focal point),

$$
\begin{aligned}
\frac{d U S_{1}(t)}{d t}= & -\beta U S_{1}(t)\left(U I_{1}(t)+A I_{1}(t)\right)\left(1-\alpha A_{1}(t)\right) \\
& -\alpha U S_{1}(t) A_{1}(t)\left(1-\beta\left(U I_{1}(t)+A I_{1}(t)\right)\right) \\
& +\gamma U I_{1}(t) \\
\frac{d A S_{1}(t)}{d t}= & \alpha U S_{1}(t) A_{1}(t)\left(1-\beta\left(U I_{1}(t)+A I_{1}(t)\right)\right) \\
& -(1-\eta) \beta\left(\rho_{1}(t)-\left(U I_{1}(t)+A I_{1}(t)\right)\right) \\
& \times\left(U I_{1}(t)+A I_{1}(t)\right)+\gamma A I_{1}(t),
\end{aligned}
$$

$$
\begin{aligned}
\frac{d U I_{1}(t)}{d t}= & \beta U S_{1}(t)\left(U I_{1}(t)+A I_{1}(t)\right)\left(1-\alpha A_{1}(t)\right) \\
& -\gamma U I_{1}(t)
\end{aligned}
$$$$
\frac{d A I_{1}(t)}{d t}=\left(\sum_{i \in N, i>1} m A I_{i}(t)-m A I_{1}(t)\right)
$$$$
+(1-\eta) \beta\left(\rho_{1}(t)-\left(U I_{1}(t)+A I_{1}(t)\right)\right)
$$$$
\times\left(U I_{1}(t)+A I_{1}(t)\right)-\gamma A I_{1}(t)
$$

For $k>1$,

$$
\begin{aligned}
\frac{d U S_{k}(t)}{d t}= & -\beta U S_{k}(t)\left(U I_{k}(t)+A I_{k}(t)\right)\left(1-\alpha A_{k}(t)\right) \\
& -\alpha U S_{k}(t) A_{k}(t)\left(1-\beta\left(U I_{k}(t)+A I_{k}(t)\right)\right) \\
& +\gamma U I_{k}(t),
\end{aligned}
$$

$$
\begin{aligned}
\frac{d A S_{k}(t)}{d t}= & \alpha U S_{k}(t) A_{k}(t)\left(1-\beta\left(U I_{k}(t)+A I_{k}(t)\right)\right) \\
& -(1-\eta) \beta\left(\rho_{k}(t)-\left(U I_{k}(t)+A I_{k}(t)\right)\right) \\
& \times\left(U I_{k}(t)+A I_{k}(t)\right)+\gamma A I_{k}(t)
\end{aligned}
$$

$$
\begin{aligned}
\frac{d U I_{k}(t)}{d t}= & \beta U S_{k}(t)\left(U I_{k}(t)+A I_{k}(t)\right)\left(1-\alpha A_{k}(t)\right) \\
& -\gamma U I_{k}(t)
\end{aligned}
$$

$$
\begin{aligned}
\frac{d A I_{k}(t)}{d t}= & \left(\frac{1}{N-1} m A I_{1}(t)-m A I_{k}(t)\right) \\
& +(1-\eta) \beta\left(\rho_{k}(t)-\left(U I_{k}(t)+A I_{k}(t)\right)\right) \\
& \times\left(U I_{k}(t)+A I_{k}(t)\right)-\gamma A I_{k}(t)
\end{aligned}
$$




\subsection{Cycle graph}

A graph with closed chain of all nodes are connected by a single cycle with binary connection is termed cycle graph (see Fig. 2b). In cycle graph, every nodes have similar two links with their neighbor nodes and the number of nodes and links are same. The dynamics can be depicted by,

\section{Subpopulation model}

$$
\frac{d \rho_{k}(t)}{d t}=\frac{1}{2} m \rho_{k-1}(t)+\frac{1}{2} m \rho_{k+1}(t)-m \rho_{k}(t),
$$

\section{SIS-UA model}

$$
\begin{aligned}
\frac{d U S_{k}(t)}{d t}= & -\beta U S_{k}(t)\left(U I_{k}(t)+A I_{k}(t)\right)\left(1-\alpha A_{k}(t)\right) \\
& -\alpha U S_{k}(t) A_{k}(t)\left(1-\beta\left(U I_{k}(t)+A I_{k}(t)\right)\right) \\
& +\gamma U I_{k}(t),
\end{aligned}
$$

$$
\begin{aligned}
\frac{d A S_{k}(t)}{d t}= & \alpha U S_{k}(t) A_{k}(t)\left(1-\beta\left(U I_{k}(t)+A I_{k}(t)\right)\right) \\
& -(1-\eta) \beta\left(\rho_{k}(t)-\left(U I_{k}(t)+A I_{k}(t)\right)\right) \\
& \times\left(U I_{k}(t)+A I_{k}(t)\right)+\gamma A I_{k}(t),
\end{aligned}
$$

$$
\begin{aligned}
\frac{d U I_{k}(t)}{d t}= & \beta U S_{k}(t)\left(U I_{k}(t)+A I_{k}(t)\right)\left(1-\alpha A_{k}(t)\right) \\
& -\gamma U I_{k}(t)
\end{aligned}
$$

$$
\begin{aligned}
\frac{d A I_{k}(t)}{d t}= & \left(\frac{1}{2} m A I_{k-1}(t)+\frac{1}{2} m A I_{k+1}(t)-m A I_{k}(t)\right) \\
+ & (1-\eta) \beta\left(\rho_{k}(t)-\left(U I_{k}(t)+A I_{k}(t)\right)\right) \\
\times & \left(U I_{k}(t)+A I_{k}(t)\right)-\gamma A I_{k}(t) \\
& A_{k}(t)=A S_{k}(t)+A I_{k}(t) .
\end{aligned}
$$

Note that, if, $k=1$, we should presume; $\rho_{0}(t)=\rho_{N}(t)$, $A I_{0}(t)=A I_{N}(t)$, while, if $k=\mathrm{N}, \rho_{N+1}(t)=\rho_{1}(t)$, $A I_{N+1}(t)=A I_{1}(t)$ should be given.

\subsection{Wheel graphs}

In graph theory, a wheel graph is formed by connecting a hub node with all nodes like star graph and each node other than hub node are connecting with their neighbor by a closed chain same as cycle graph (Fig. 2c). Hub node has $N-1$ link with neighbor and others nodes have three connection with hub and neighbor nodes for $N$ nodes graphs. The total number of links are $2(N-1)$. The SIS-UA metapopulation model for wheel graph is below.

\section{Subpopulation model}

$$
\frac{d \rho_{1}(t)}{d t}=\sum_{i \in N, i>1} \frac{1}{3} m \rho_{i}(t)-m \rho_{1}(t), \quad k=1
$$

$$
\begin{aligned}
\frac{d \rho_{k}(t)}{d t}= & \frac{1}{N-1} m \rho_{1}(t)+\frac{1}{3} m \rho_{k-1}(t) \\
& +\frac{1}{3} m \rho_{k+1}(t)-m \rho_{k}(t), \\
k> & 1, k=2,3,4, \ldots N .
\end{aligned}
$$

\section{SIS-UA model}

For $k=1$ (focal point),

$$
\begin{aligned}
\frac{d U S_{1}(t)}{d t}= & -\beta U S_{1}(t)\left(U I_{1}(t)+A I_{1}(t)\right)\left(1-\alpha A_{1}(t)\right) \\
& -\alpha U S_{1}(t) A_{1}(t)\left(1-\beta\left(U I_{1}(t)+A I_{1}(t)\right)\right) \\
& +\gamma U I_{1}(t), \\
\frac{d A S_{1}(t)}{d t}= & \alpha U S_{1}(t) A_{1}(t)\left(1-\beta\left(U I_{1}(t)+A I_{1}(t)\right)\right) \\
& -(1-\eta) \beta\left(\rho_{1}(t)-\left(U I_{1}(t)+A I_{1}(t)\right)\right) \\
& \times\left(U I_{1}(t)+A I_{1}(t)\right)+\gamma A I_{1}(t), \\
\frac{d U I_{1}(t)}{d t}= & \beta U S_{1}(t)\left(U I_{1}(t)+A I_{1}(t)\right)\left(1-\alpha A_{1}(t)\right) \\
& -\gamma U I_{1}(t), \\
\frac{d A I_{1}(t)}{d t}= & \left(\sum_{i \in N, i>1} \frac{1}{3} m A I_{i}(t)-m A I_{1}(t)\right) \\
& +(1-\eta) \beta\left(\rho_{1}(t)-\left(U I_{1}(t)+A I_{1}(t)\right)\right) \\
& \times\left(U I_{1}(t)+A I_{1}(t)\right)-\gamma A I_{1}(t) .
\end{aligned}
$$

For $k>1$,

$$
\begin{aligned}
\frac{d U S_{k}(t)}{d t}= & -\beta U S_{k}(t)\left(U I_{k}(t)+A I_{k}(t)\right)\left(1-\alpha A_{k}(t)\right) \\
& -\alpha U S_{k}(t) A_{k}(t)\left(1-\beta\left(U I_{k}(t)+A I_{k}(t)\right)\right) \\
& +\gamma U I_{k}(t), \\
\frac{d A S_{k}(t)}{d t}= & \alpha U S_{k}(t) A_{k}(t)\left(1-\beta\left(U I_{k}(t)+A I_{k}(t)\right)\right) \\
& -(1-\eta) \beta A S_{k}(t)\left(U I_{k}(t)+A I_{k}(t)\right) \\
& +\gamma A I_{k}(t), \\
\frac{d U I_{k}(t)}{d t}= & \beta U S_{k}(t)\left(U I_{k}(t)+A I_{k}(t)\right)\left(1-\alpha A_{k}(t)\right) \\
& -\gamma U I_{k}(t), \\
\frac{d A I_{k}(t)}{d t}= & \frac{1}{N-1} m A I_{1}(t)-m A I_{k}(t)+\frac{1}{3} m A I_{k+1}(t) \\
+ & \left.\frac{1}{3} m A I_{k-1}(t)\right)+(1-\eta) \beta\left(\rho_{k}(t)\right. \\
- & \left.\left(U I_{k}(t)+A I_{k}(t)\right)\right)\left(U I_{k}(t)+A I_{k}(t)\right) \\
- & \gamma A I_{k}(t), \\
& A A_{k}(t)=A S_{k}(t)+A I_{k}(t) .
\end{aligned}
$$

If, $k=2, \rho_{k-1}(t)=\rho_{N}(t), A I_{k-1}(t)=A I_{N}(t)$, and, $k=\mathrm{N}, \rho_{N+1}(t)=\rho_{2}(t), A I_{N+1}(t)=A I_{2}(t)$. 


\subsection{Complete graph}

A complete graph in the field of graph theory is a graph where every node relates to each other (see Fig. 2d). For $N$ nodes complete graph, the number of edges are $N(N-1) / 2$. The SIS-UA epidemic model for complete graph is below

\section{Subpopulation model}

$$
\frac{d \rho_{k}(t)}{d t}=\sum_{i \in N, i \neq k} \frac{1}{N-1} m \rho_{i}(t)-m \rho_{k}(t),
$$

\section{SIS-UA model}

$$
\begin{aligned}
\frac{d U S_{k}(t)}{d t}= & -\beta U S_{k}(t)\left(U I_{k}(t)+A I_{k}(t)\right)\left(1-\alpha A_{k}(t)\right) \\
& -\alpha U S_{k}(t) A_{k}(t)\left(1-\beta\left(U I_{k}(t)+A I_{k}(t)\right)\right) \\
& +\gamma U I_{k}(t), \\
\frac{d A S_{k}(t)}{d t}= & \alpha U S_{k}(t) A_{k}(t)\left(1-\beta\left(U I_{k}(t)+A I_{k}(t)\right)\right) \\
& -(1-\eta) \beta A S_{k}(t)\left(U I_{k}(t)+A I_{k}(t)\right) \\
& +\gamma A I_{k}(t), \\
\frac{d U I_{k}(t)}{d t}= & \beta U S_{k}(t)\left(U I_{k}(t)+A I_{k}(t)\right)\left(1-\alpha A_{k}(t)\right) \\
& -\gamma U I_{k}(t), \\
\frac{d A I_{k}(t)}{d t}= & \left(\sum_{i \in N_{i \neq k}} \frac{1}{N-1} m A I_{i}(t)-m A I_{k}(t)\right) \\
& +(1-\eta) \beta\left(\rho_{k}(t)-\left(U I_{k}(t)+A I_{k}(t)\right)\right) \\
& \times\left(U I_{k}(t)+A I_{k}(t)\right)-\gamma A I_{k}(t), \\
& A_{k}(t)=A S_{k}(t)+A I_{k}(t) .
\end{aligned}
$$

Following the above development of equations, to put it another way, we consider the total six different scenarios to understand the effect of awareness and migration in all possible cases (Appendix). Namely, Case 1 (Eqs. (1)-(34)), we consider only aware infected individuals can migrate. In Case 2 (Appendix, Eqs. (A.1)-(A.12)), aware susceptible and aware infected both can migrate. In Case 3 (Appendix, Eqs. (A.13)-(A.36)), all people both aware and unaware for susceptible and infected can migrate. Defaults case is considered for Case 4, where no migration occurred $(m=0)$. In Case 5 (Appendix, Eqs. (A.37)-(A.42)), only aware susceptible individuals can migrate. Finally, only unaware infected can migrate is illustrated in Case 6 (Appendix, Eqs. (A.43)-(A.48)).

Now, we have accomplished to establish all the analytical frameworks for $N$ nodes, where, $k=1,2,3, \ldots, N$. $k$ is the number of nodes (subpopulation) and $N$ is the maximum number of nodes. The number of all aware individuals is denoted by $A_{k}(t)$ and all the parameters: $\beta, \eta, \gamma$ and $\alpha$ are considered positive constants. At the

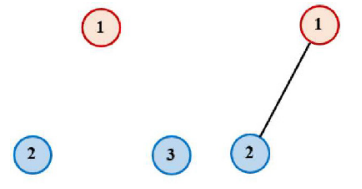

(a)

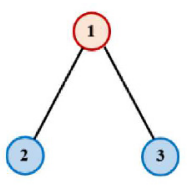

(c)

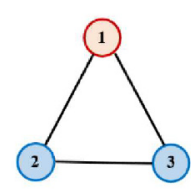

(d)
Fig. 3. Schematic image of the metapopulation SIS-UA model composed of three nodes (subpopulations). (a) All nodes are disconnected from each other, (b) only node 1 and node 2 are connected but node 3 is isolated, (c) node 1 is connected with node 2 and node 3 , but node 2 and node 3 are disconnected. (d) All nodes are connected with each others.

beginning, we presumed some set of initial values, $\rho_{k}(0)=$ $U S_{k}(0) \approx 1, A S_{k}=0, U I_{k}(0)=0$ and $A I_{k}(0) \approx 0$. Thus, the above set of equations can be solved numerically by means of the explicit finite difference method.

\section{Results and discussion}

The metapopulation epidemic model with the impact of awareness effect can assist individuals to decrease their threat of being infected in a population. This approach is an essential mathematical framework used in population ecology, environment, and government policy to explain the epidemic dynamics for different spatial graphs structure by a highly fragmented location in which the individuals are organized in isolated discrete patches (subpopulations). In current work, different cases are explored to describe the awareness effect with epidemic spreading in a metapopulation modeling approach to display how accurately described the migration patterns. Numerical analysis with depth exploration is inspected to display how well the proposed framework may be applied in practice. At first, the effect of infected individuals is depicted for both aware and unaware situation for node $N=3$, which can be thought specific and fundamental configuration. The full phase diagrams are illustrated to explain the various cases and default case by considering the possibility of migrated individuals for the node, $N=7$. Finally, the epidemic model is discussed to extend the number of node (up to $N=51$ ) by taking four different graphs where the only aware infected individual can travel.

\subsection{Dynamics of three subpopulation $(N=3)$}

Let us presume that there are three nodes $N=3$; nodes are numbered 1, 2 and 3 shown in Figure 3 by considering four cases. In the first case; case (a) (Fig. 3a), three nodes are isolated from each other where the individuals in each node never walk to other nodes. In case (b) of Figure 3b, node 3 is isolated from nodes 1 and 2, only individuals from nodes 1 and 2 migrate by a random walk. In Figure 3c, node 1 is connected with both 2 and 3, individuals can go back and forth between node 1 to node 2 and node 1 to node 3 by random walk, but no link between node 2 and node 3 in case (c). Finally, in case (d), all nodes are connected to each other, individuals can move from any nodes by random walks in Figure 3d. 


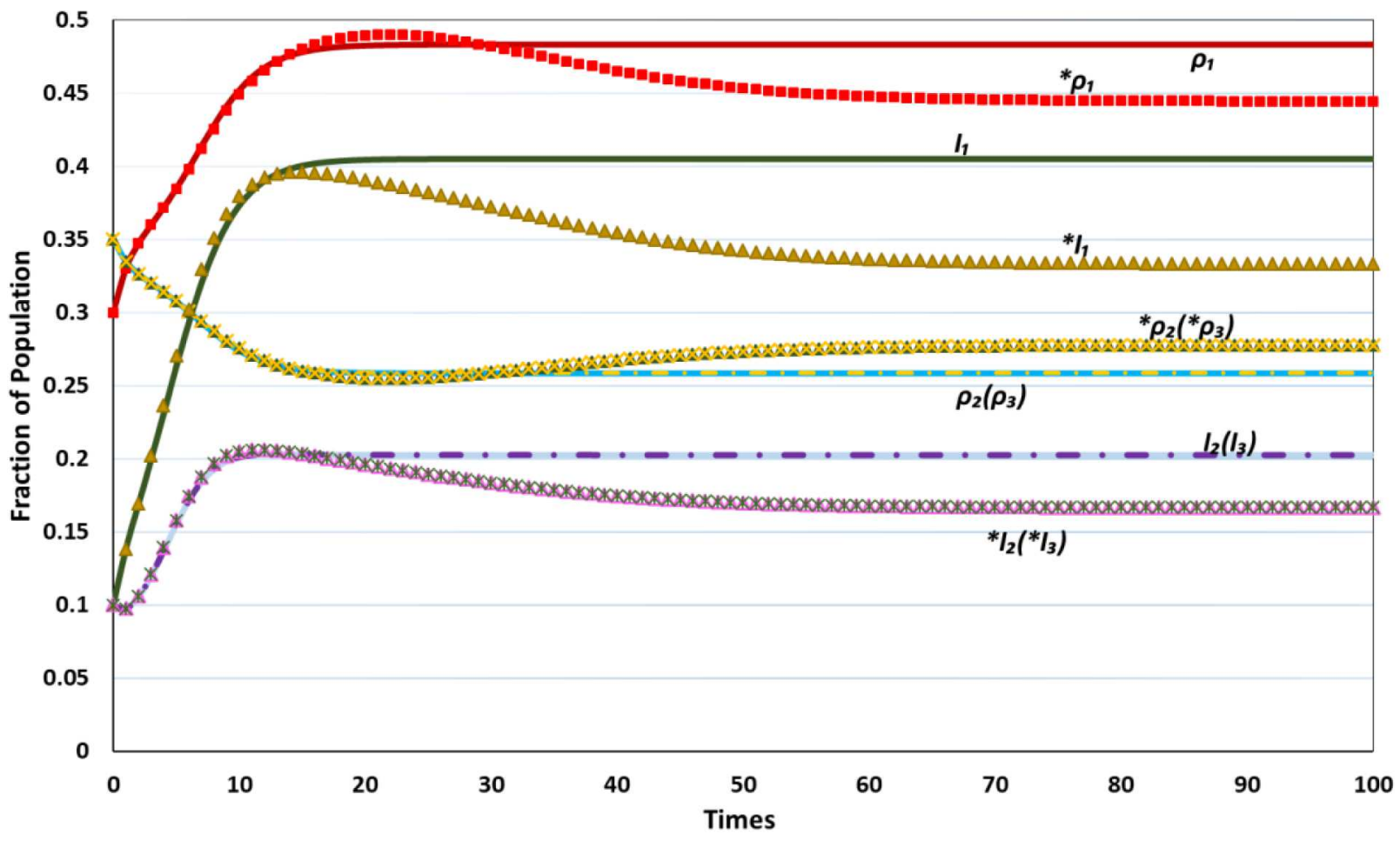

Fig. 4. Results for the case (c) (Fig. 3c), plots of infected individuals and island population for each subpopulation are displayed against times at infection rate $\beta=1.0$ and recovery rate $\gamma=0.1$ for initial values $\rho_{1}(0)=0.3, \rho_{2}(0)=\rho_{3}(0)=0.35$, $* \rho_{1}(0)=0.3, * \rho_{2}(0)=* \rho_{3}(0)=0.35, I_{1}(0)=I_{2}(0)=I_{3}(0)=* I_{1}(0)=* I_{2}(0)=* I_{3}(0)=0.1$. The dot lines indicate the aware situation and plain lines show the unaware condition.

An improved SIS-UA epidemic model with metapopulation migration model between three nodes are modeled for four different cases in Figure 3. In numerical simulation for $N=3$, the infection rate $\beta=1.0$, recovery rate $\gamma=0.1$ are considered for initial condition; $\rho_{1}(0)=0.3, \rho_{2}(0)=$ $\rho_{3}(0)=0.35, A I_{1}(0)=A I_{2}(0)=A I_{3}(0)=0.1$.

Firstly, case (c) is explored to observe the time evolution of the subpopulation densities and infection densities (unaware and aware) in each node, where node 1 is connected with both node 2 and 3 , but 2 and 3 are separated. In Figure 4, the plain lines denote the fraction of population presuming no information spreading; $\alpha=0.0$, whereas the dot lines (plots) indicate the fractions of the population for the effect of awareness $(\alpha=0.4)$. Red, blue and yellow plain lines indicate the fraction of population in node 1,2 and $3\left(\rho_{1}, \rho_{2}, \rho_{3}\right)$ for the unaware state, whereas, red, blue and yellow dots line indicate the aware state of a subpopulation $\left(* \rho_{1}, * \rho_{2}, * \rho_{3}\right)$. Similarly, green, violate and light blue for both dot and plain lines show the infected individuals $\left(I_{1}, I_{2}, I_{3}, * I_{1}, * I_{2}, * I_{3}\right)$ for node 1,2 and 3 . Let alone, when we refer to the graph configuration of the case (c), the time evolution of subpopulation and infected individuals in node 3 is consistent with node 2 , because the initial densities in node 2 and 3 are same. At the steady state, the subpopulation and infected individuals in each node approach the constant values. Furthermore, for without awareness, the graph of the subpopulation and infected individuals depicted in Figure 4 is validated with Nagatani et al. [13] work. With this intention, dot lines clearly show the significant effect of information in metapopulation for the random walk.

In Figure 5, the four cases; depending on graph configuration given in Figure 3, are compared when we are concerned on respective time-series values at equilibrium. Here, Figure 3a compares densities of a subpopulation, and Figure 3b compares infected individuals for both unaware and aware situation. Again let us confirm this case (Case 1) allows that only aware infected individuals can migrate. Based on the connection among nodes, case (a) has no change of densities from the initial values discussed above for node 1,2 and 3 because all the nodes are isolated from each other. In case (b), shows a very small change of population in both node 1 and node 2 , due to only one connection between node 1 and 2 exist. On the other hand, case (c) displays the highest variation where the population of node 1 (both cases) have maximum densities and node 2 and 3 have the lowest densities. In view of the case (c); star graph, being representative of the heterogeneous graph, the focal node seems to be supplying source of disease. Finally, all subpopulations have an equal situation at equilibrium in case (d), where all the nodes are connected with each other allowing heavy traffic of (aware) infected individuals.

The fraction of infected individuals for four cases are compared in Figure 5b, here, $I_{1}, I_{2}, I_{3}, * I_{1}, * I_{2}$ and $* I_{3}$ denote the infected individuals in each subpopulation at steady state. The sum of infected individuals in each subpopulation is signified by $I_{\rho}=I_{1}+I_{2}+I_{3}$ for 


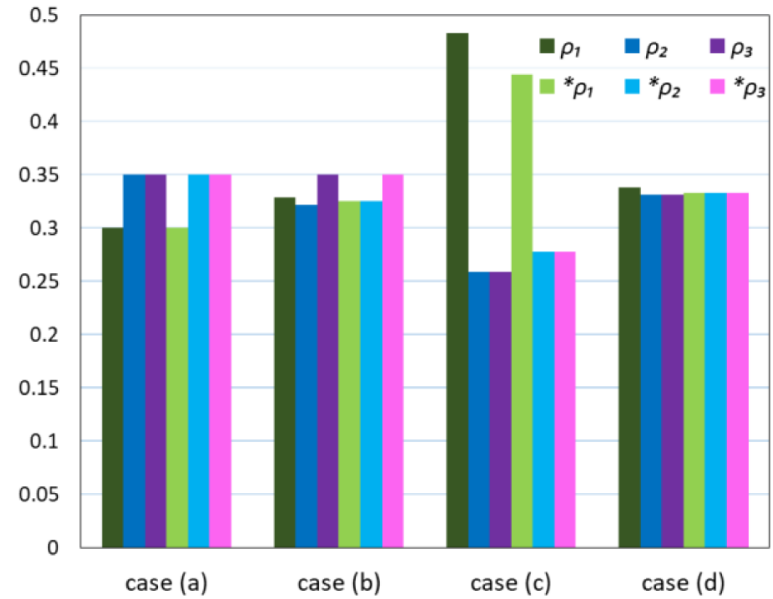

(a)

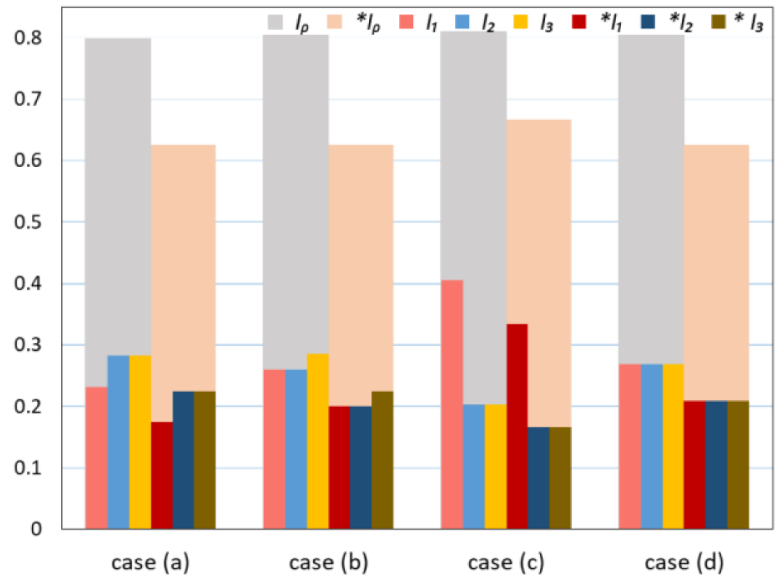

(b)

Fig. 5. The bar graph depicts (a) the total individuals of each subpopulation for both unaware and aware states (b) the total infected individuals for both unaware and aware states. Case (a), all subpopulation are isolated, case (b)2, node 1 and node 2 are connected, case (c), node 1 is connected with node 2 and node 3 , but node 2 and 3 are disconnected and in case (d) all nodes are connected with each others are presented for $N=3, \beta=1.0$ and $\gamma=0.1$.

unaware state and $* I_{\rho}=* I_{1}+* I_{2}+* I_{3}$ for the aware situation. It is clear from Figure $5 \mathrm{~b}$ that, in all cases, the aware infected individual in each subpopulation is always smaller than unaware infected people that strongly support the hypothesis of information spreading in the epidemic model. Meanwhile, case (c) shows the largest fraction of aware infected vis-a-vis other three cases, perhaps because case (c) has extremely heterogeneous topology amid those. In case (d), at equilibrium, the impact of awareness can be observed clearly by comparing both summing bars for the unaware and aware situation, although the infected individuals in respective nodes are equal. Based on this assumption, we consider this equilibrium condition throughout this work to signify the impact of awareness in metapopulation epidemic model.

\subsection{Dynamics of seven subpopulation $(N=7)$}

Motivated by the above discussion, we present a thorough analysis on the behavior of infected individuals in metapopulation models for seven nodes in four different types of graph: star, cycle, wheel and complete by considering six cases are given below.

Case 1. Only aware infected individuals can migrate (Fig. 6(i-*)).

Case 2. Both aware susceptible and aware infected can migrate (Fig. 6(ii-*)).

Case 3. Any people both aware and unaware for susceptible and infected can migrate (Fig. 6(iii-*)).

Case 4. No migration is occurring, all nodes are isolated (Fig. $\left.7\left(\mathrm{i}^{*}\right)\right)$.

Case 5. Only aware susceptible individuals can migrate (Fig. 7(ii-*)).
Case 6. Only unaware infected can migrate (Fig. 7(iii-*)).

Here, aside from the case 4 , rest of the cases individual can migrate based on the subpopulation model, whereas, respected individuals for each case, those who are committed to migrate can follow the SIS-UA epidemic model.

It is revealed from Figures 6 and 7 , the effect of awareness is showed in metapopulation epidemic model for four different graphs of $N=7$ (subpopulation) with respect to the disease transmission rate $\beta$ and the information spreading rate $\alpha$. Moreover, the subpopulation in metapopulation is represented by the star graphs in Figures $6\left(^{*}-\mathrm{a}\right)$ and $7\left(^{*}-\mathrm{a}\right)$, the cycle graphs in Figures $6\left(*_{-} \mathrm{b}\right)$ and $7\left(*_{-} \mathrm{b}\right)$, the wheel graphs in Figures $6\left(*^{*} \mathrm{c}\right)$ and $7\left(*_{-c}\right)$ and the complete graphs in Figures $6\left(*^{*}-\mathrm{d}\right)$ and $7\left(*^{*}-\mathrm{d}\right)$. Here, the infected individuals $I_{\text {total }}$ is taken as the sum of all infected individuals (both unaware and aware) accumulated seven nodes at equilibrium.

In general, because an increase of $\alpha$ ensures less size of infection at a same $\beta$ irrespective to Cases as well as subpopulation topology, there have been confirmed the substantial effect of awareness to lessen the spreading of infected individuals in a population.

Now, by comparing four graphs: star, cycle, wheel and complete, a monotonic decreasing tendency in this order is observed for all Cases except Case 4 (Fig. 7(i-*)) since there are existing extremity nodes in case of a star graph. It also illustrates from displayed figures that the network structure of the complete graph can reduce the threat of infection considerably compare with others graphs because every node is connected each other, which ensures a migrant, regardless being infected or not, has the maximal number of heading subpopulations.

The effect depicted by different graphs in Figure $7\left(\right.$ ii- $\left.^{*}\right)$ under the setting of Case 5 results from how only aware susceptible individuals migrating through four topologies oppresses disease spreading. Expectedly, the higher 


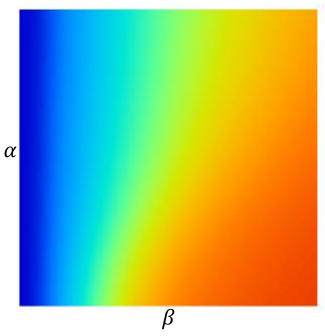

(i-a)

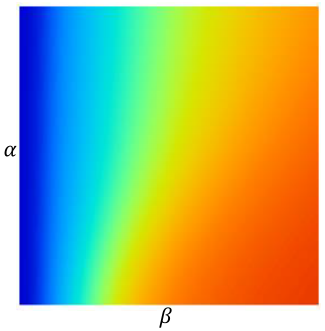

(ii-a)

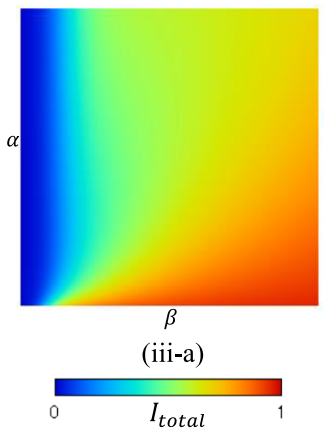

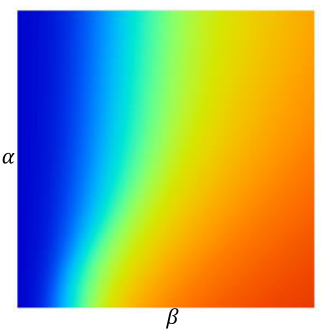

(i-b)

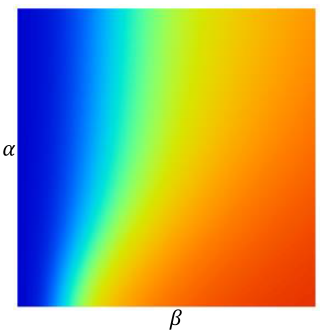

(ii-b)

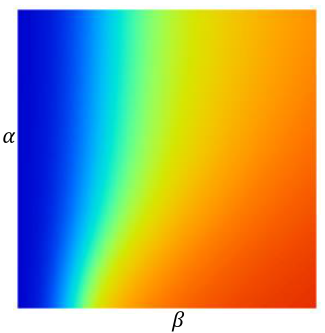

(iii-b)

$I_{\text {total }}$

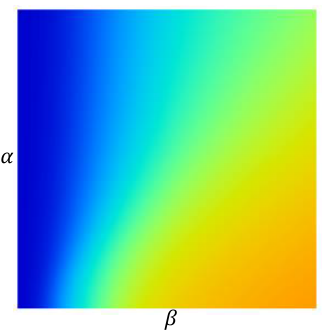

(i-c)

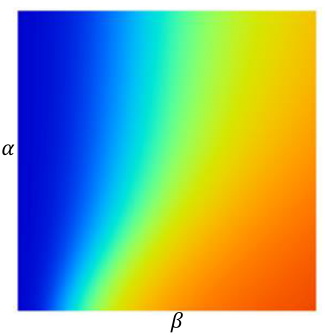

(ii-c)

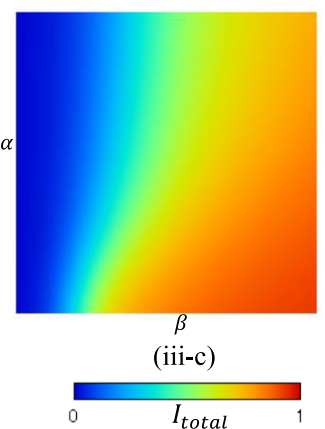

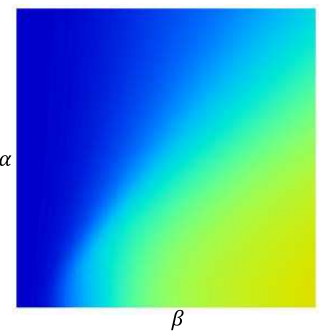

(i-d)

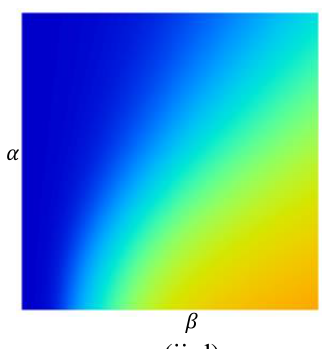

(ii-d)

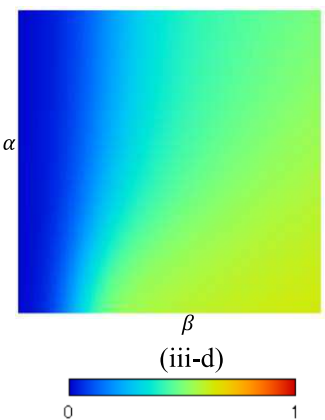

Fig. 6. The $2 \mathrm{D}$ full phase diagram for the disease transmission rate and the information spreading rate at equilibrium for Case 1 (i- $\left.^{*}\right)$, Case 2 (ii- $^{*}$ ) and Case 3 (iii- $\left.{ }^{*}\right)$ are presented for $N=7$. The first column display the star graph (*-a), second column, cycle graph $\left(*_{-}^{*}\right)$, third column, wheel column $\left(*^{*}\right.$ c) and the last column show the complete graph $\left(*_{-}^{*}\right)$. Here, the parameters are considered: $\gamma=0.1, \eta=0.3$ and $m=0.2$.

information spreading rate $\alpha$ accelerates the migration process because only aware susceptible can be mobile. Except for star graph (Fig. 7(ii-a)), other graphs can be lessened the infection for higher $\alpha$. This tendency comes from the following mechanism. Referring to Figure 5, other topologies than star graph, the hub, and peripheral nodes has comparable population densities, whereas, in star graph, the population density of hub node is much higher than that of peripherals. This fact ensures that other topologies than star graph enjoy the advantage of information effect to oppress disease spreading because peripherals, as well as hub nodes, have a higher fraction on aware people. On the other hand, the star graph makes migrating people, who are aware and susceptible, be concentrated in the focal node. Thus relatively, less fraction of aware people in the peripherals. This inevitably makes less sensitivity along $\alpha$ be observed.

However, in Figure 7(iii-*) for Case 6, where only unaware infected can migrate, display exactly opposite incidence liken with other graphs, the final epidemic size is higher for larger values of the information spreading rate. Meanwhile, observing to quantitatively compare from Case 1 to Case 6, we note that the number of infected individuals in Case 6 with presuming complete graph where only unaware infected can migrate shows smallest. Yet, other cases than complete graph are not so significant to oppress the disease.

Therefore, in a word, awareness can suppress disease spreading which depend on individual's choice by taking protection or migration. Although the mitigation by an unaware infected individual makes the awareness effect backed by information spreading be an invisible and little bit counterproductive as opposed to other cases, interestingly and suggestively, this scheme can reduce the infected thread most remarkably as long as the complete graph is presumed. It can imply that some sort of quarantine policy the government taking or forceful social isolation keeping inappropriate people away may contain some rationality to make remaining people safe. But it is only true when there are a sufficient number of isolated sites, otherwise, the policy does not work so effectively.

\subsection{Dynamics of many subpopulations}

To clarify the effect of higher-order nodes (up to $N=51$ ) in metapopulation SIS-UA epidemic model, Figure 8 displays the effect of total infected individuals at equilibrium. 


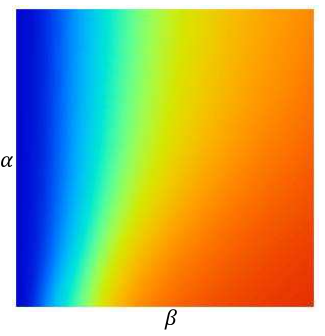

(i-a)

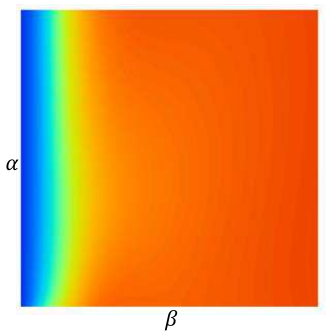

(ii-a)

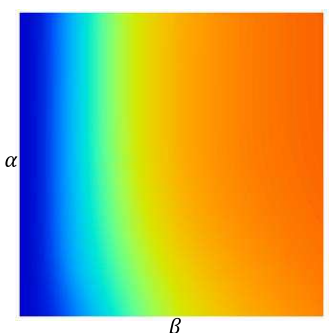

(iii-a)

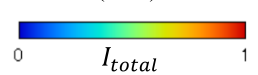

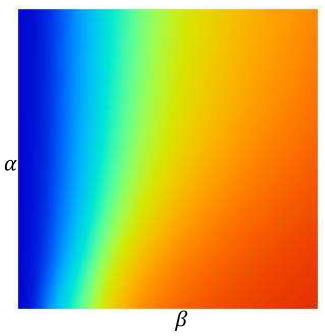

(i-b)

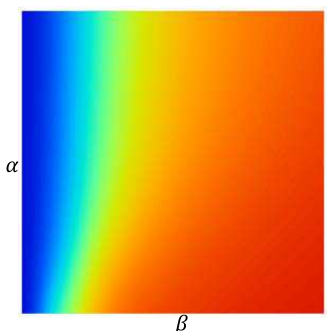

(ii-b)

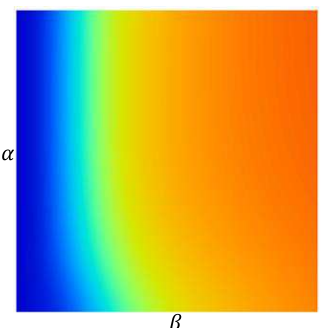

(iii-b)

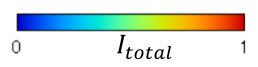

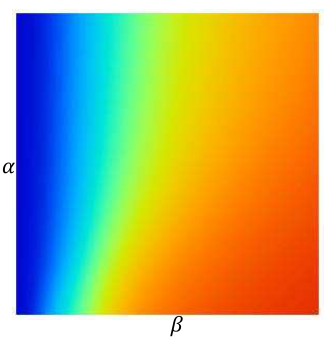

$(\mathrm{i}-\mathrm{c})$

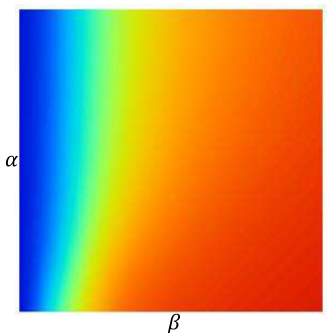

(ii-c)

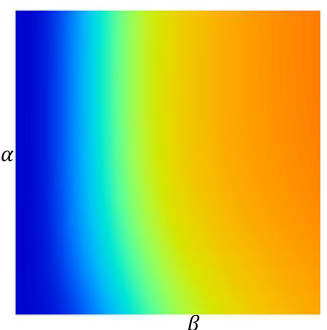

(iii-c)

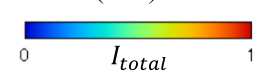

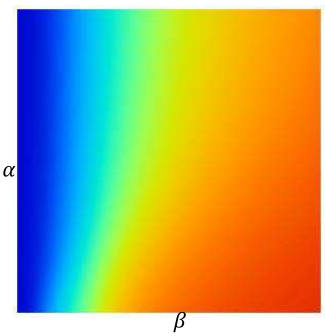

(i-d)

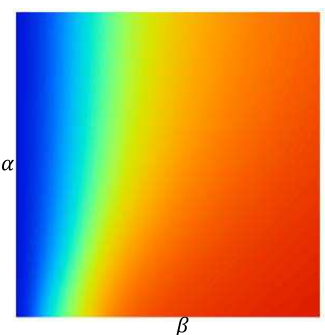

(ii-d)

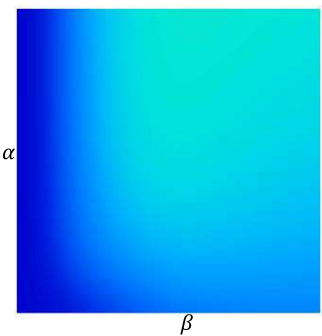

(iii-d)

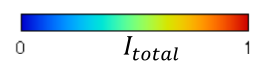

Fig. 7. The $2 \mathrm{D}$ full phase diagram for the disease transmission rate and the information spreading rate at equilibrium for Case $4\left(\right.$ i- $\left.^{*}\right)$, Case $5\left(\right.$ ii- $\left.^{*}\right)$ and Case $6\left(\right.$ iii- $\left.^{*}\right)$ are presented for $N=7$. In the first column, the star graph $(*$-a), second column, cycle graph $\left(*_{-}\right.$b), third column, wheel column $\left(*_{-}\right.$) $)$and the last column, the complete graph $(*-d)$ are presented. Here, the parameters are considered: $\gamma=0.1, \eta=0.3$ and $m=0.2$.

In Figure 8, four topologies: star, cycle, wheel and complete are compared for total number of nodes; $1,3,5,7$, $11,21,21,31,41$ and 51 display the influence of total infected individuals when presuming Case 1, where only aware infected individuals can migrate. Comparing four lines, it can be concluded that the infection densities at equilibrium for the complete graph are obviously lower than others, and more importantly, of which tendency becomes more evident with the increase of degree. It is because the number of links each node has increased with the increase of the number of nodes, which ensures many alternative headings for aware infected migrants. However, star and cycle show almost the same densities of infected individuals for higher nodes due to the connection for each node is one and two. Wheel graph displays the superior final epidemic size (say, showing lower densities) to star and cycle but much higher densities than a complete graph. As long as a sufficient number of nodes is presumed, the order of performance to oppress the final epidemic size is the same as the order of the total number of links. In a nutshell, complete graph, having the maximal number of links, quantified by $N(N-1)$, shows the best performance.

\section{Conclusion}

In the current paper, the impact of information spreading on metapopulation epidemic model in a network population is theoretically estimated and analyzed. The intention of present work is to develop a metapopulation migration model on the star, cycle, wheel and complete graphs on three, seven and $N$ nodes as a prototype for the complex network. The mathematical framework based on mean field approximation with two-layer compartment model derived from SIS-UA is newly established and carried out numerical simulations to validate the model. Hence, four variant models are implemented to investigate the effect of aware and unaware on metapopulation epidemic SIS model for six different cases by a random walk. The effect of awareness, infection, self-protection, migration and spatial networking structure are shown clearly for time series graphs and by portraying full phase diagram. Firstly, from the time evaluation graphs, it can be concluded that the information about the disease before illness can reduce the number of infected individuals phenomenally by encouraging people to take self-protection and go save place. Secondly, from the full phase diagram, 


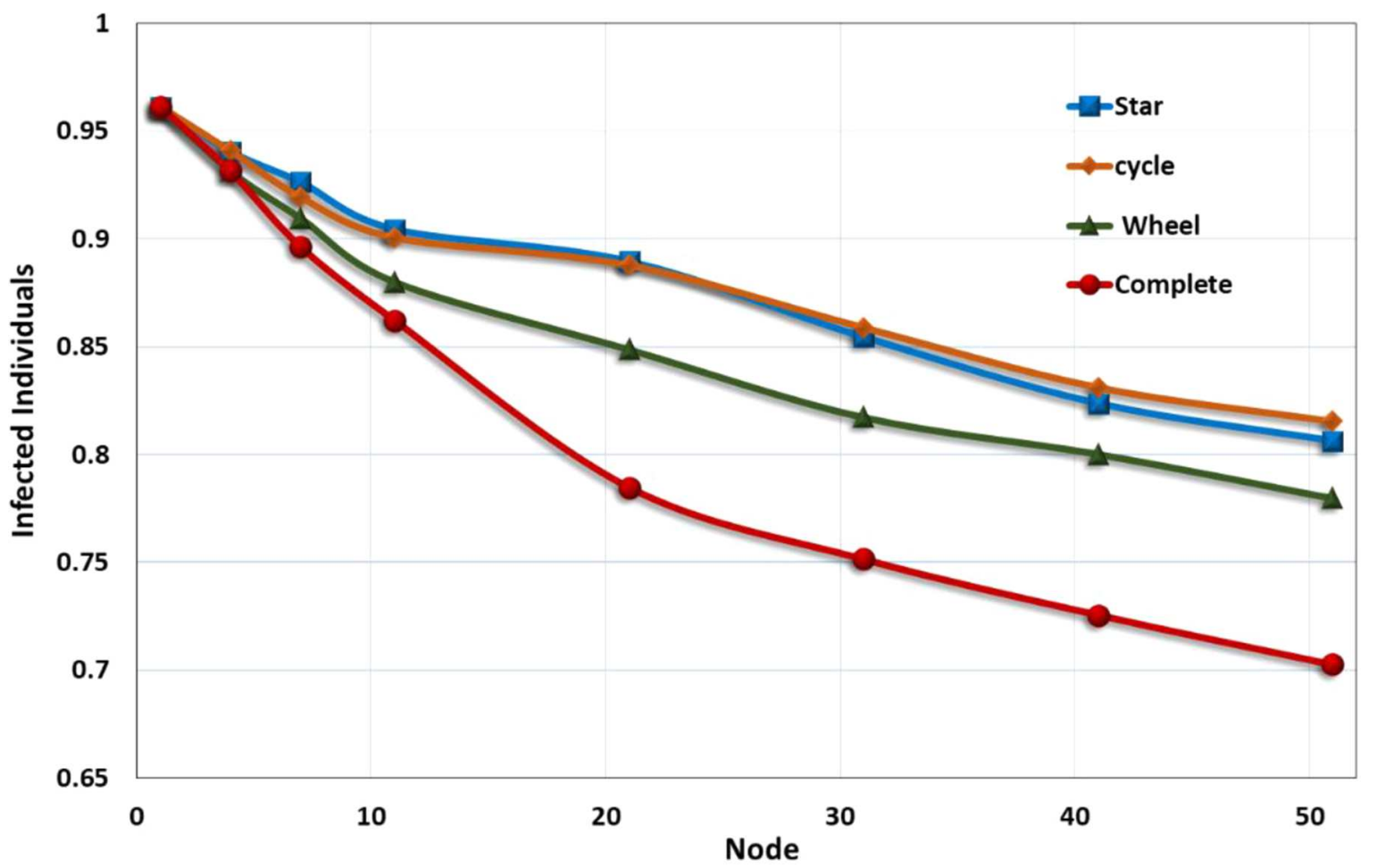

Fig. 8. Line graph of total infected individuals against subpopulation number on star, cycle, wheel and complete graph at steady state situation are considered, where node (subpopulation) $N=1,5,7,11,21,31,41$ and 51 .

the complete graph shows a better networking structure in metapopulation model to reduce diseases. Moreover, at the point of individual choice, the infected individuals reduced remarkably when only aware infected can migrate. On the other hand, at the point of authority or government standpoint, if unaware people are migrated by forcefully then the spreading of infection can reduce remarkably. Finally, it is revealed from the study of a higher node, the complete graph displays the most significant tendency to suppress the infected individuals in an epidemic compare with the star, cycle and wheel graph. At the point of individual's choice, the complete graph for Case 1 shows the significant reduction of disease, but, in Case 6 displays superior reduction of infected individuals. Furthermore, as the most important finding here, in the proposed framework, the effect of awareness on the spreading of infection can reduce the disease diffusion remarkably for all cases, because the awareness can promote people to take self-protection or move in a safe place.

In the current work, we presumed constant migration rate, $m$ to meet with the most fundamental idea of random walk the pioneer study took [13]. In practice and in reality, the driving force letting people evacuate (or, as the responsible body, government takes quarantine policy) should be dependent on the current infectious fraction at a destination site. Such a complex situation obviously containing non-linear effects will be explored in our future studies.

This study was partially supported by Grant-in-Aid for Scientific Research from JSPS, Japan, KAKENHI (Grant No.
18K18924) and Kakihara Foundation awarded to Professor Tanimoto. We would like to express our gratitude to them.

\section{Author contribution statement}

K.M. Ariful Kabir and Jun Tanimoto designed and performed the research as well as wrote the paper.

\section{References}

1. W.O. Kermack, A.G. McKendrick, Proc. R. Soc. Lond. A 115, 700 (1927)

2. I. Hanski, Nature 396, 5 (1998)

3. M. Bonaventura, V. Nicosia, V. Latora, Phys. Rev. E 89, 12803 (2014)

4. S. Meloni, N. Perra, A. Arenas, S. Gómez, Y. Moreno, A. Vespignani, Sci. Rep. 1, 62 (2011)

5. A. Buscarino, L. Fortuna, M. Frasca, V. Latora, Europhys. Lett. 82, 38002 (2008)

6. T. Nagatani, G. Ichinose, K. Tainaka, J. Phys. Soc. Jpn. 86, 113001 (2017)

7. M. Draief, A. Ganesh, Discrete Event Dyn. Syst. 21, 41 (2011)

8. J. Peterson, Stochastic Processes Appl. 121, 609 (2010)

9. R. Durrett, S.A. Levin, Theor. Popul. Biol. 46, 363 (1994)

10. T.E. Harries, Ann. Probab. 2, 969 (1974)

11. C. Pu, S. Li, J. Yang, Physica A 432, 230 (2015)

12. V. Colizza, A. Vespignani, J. Theor. Biol. 251, 450 (2008)

13. T. Nagatani, G. Ichinose, K. Tainaka, J. Theor. Biol. 450, 66 (2018)

14. K.M.A. Kabir, K. Kuga, J. Tanimoto, Chaos Solitons Fractals 119, 118 (2019) 
15. K.M.A. Kabir, J. Tanimoto, Commun. Nonlinear Sci. Numer. Simul. 72, 565 (2019)

16. K.M.A. Kabir, K. Kuga, J. Tanimoto, Chaos Solitons Fractals 119, 180 (2019)

17. K. Kuga, J. Tanimoto, J. Stat. Mech.: Theory Exp. 2018, 023407 (2018)

18. A. Barrat, M. Barthélemy, A. Vespignani, Dynamical Processes on Complex Networks (Cambridge University Press, Cambridge, UK, 2008)

19. S. Kwon, Y. Kim, Phys. Rev. E 84, 012813 (2013)

20. H. Zhang, Z.H. Guan, T. Li, X.H. Zhang, D.X. Zhang, Physica A 392, 974 (2013)

21. S. Funk, M. Salathé, V.A.A. Jansen, J. R. Soc. Interface 7, $1247(2010)$
22. C. Granell, S. Gómez, A. Arenas, Phys. Rev. E 90, 012808 (2014)

23. C. Granell, S. Gómez, A. Arenas, Phys. Rev. Lett. 111, 12870 (2013)

24. W. Wang, Q.H. Liu, S. Cai, M. Tang, A. Lidia, Braunstein, H.E. Stanley, Sci. Rep. 6, 29259 (2016)

25. S. Funk, E. Gilad, C. Watkins, A. Vincent, A. Jansen, Proc. Natl. Acad. Sci. 106, 6872 (2009)

26. Q. Wu, X. Fu, M. Small, X.J. Xu, Chaos 22, 013101 (2012)

27. Y. Pan, Z. Yan, Physica A 491, 45 (2018)

28. E. Fukuda, J. Tanimoto, M. Akimoto, Chaos Solitons Fractals 80, 47 (2015)

29. A. Moinet, R. Pastor-Satorras, A. Barrat, Phys. Rev. E 97, 012313 (2018)

\section{Appendix A}

\section{A.1 Case 2}

\section{Star graphs: SIS-UA model}

For $k=1$, (Focal Point)

$$
\begin{aligned}
\frac{d A S_{1}(t)}{d t}= & \left(\sum_{i \in N, i>1} m A S_{i}(t)-m A S_{1}(t)\right)+\alpha U S_{1}(t) A_{1}(t)\left(1-\beta\left(U I_{1}(t)+A I_{1}(t)\right)\right) \\
& -(1-\eta) \beta\left(\rho_{1}(t)-\left(U I_{1}(t)+A I_{1}(t)\right)\right)+\gamma A I_{1}(t), \\
\frac{d A I_{1}(t)}{d t}= & \left(\sum_{i \in N, i>1} m A I_{i}(t)-m A I_{1}(t)\right)+(1-\eta) \beta\left(\rho_{1}(t)-\left(U I_{1}(t)+A I_{1}(t)\right)\right)\left(U I_{1}(t)+A I_{1}(t)\right) \\
& +\alpha U I_{1}(t) A_{1}(t)(1-\gamma)-\gamma A I_{1}(t),
\end{aligned}
$$

For $k>1$,

$$
\begin{aligned}
\frac{d A S_{k}(t)}{d t}= & \left(\frac{1}{N-1} m A S_{1}(t)-m A S_{k}(t)\right) \\
& +\alpha U S_{k}(t) A_{k}(t)\left(1-\beta\left(U I_{k}(t)+A I_{k}(t)\right)\right)-(1-\eta) \beta\left(\rho_{k}(t)-\left(U I_{k}(t)\right.\right. \\
& \left.\left.+A I_{k}(t)\right)\right)\left(U I_{k}(t)+A I_{k}(t)\right)+\gamma A I_{k}(t), \\
\frac{d A I_{k}(t)}{d t}= & \left(\frac{1}{N-1} m A I_{1}(t)-m A I_{k}(t)\right)+(1-\eta) \beta\left(\rho_{k}(t)-\left(U I_{k}(t)+A I_{k}(t)\right)\right)\left(U I_{k}(t)\right. \\
& \left.+A I_{k}(t)\right)+\alpha U I_{k}(t) A_{k}(t)(1-\gamma)-\gamma A I_{k}(t),
\end{aligned}
$$

\section{Cycle graph: SIS-UA model}

$$
\begin{aligned}
\frac{d A S_{k}(t)}{d t}= & \left(\frac{1}{2} m A S_{k-1}(t)+\frac{1}{2} m A S_{k+1}(t)-m A S_{k}(t)\right) \\
& +\alpha U S_{k}(t) A_{k}(t)\left(1-\beta\left(U I_{k}(t)+A I_{k}(t)\right)\right)-(1-\eta) \beta\left(\rho_{k}(t)-\left(U I_{k}(t)+A I_{k}(t)\right)\right) \\
& \times\left(U I_{k}(t)+A I_{k}(t)\right)+\gamma A I_{k}(t) \\
\frac{d A I_{k}(t)}{d t}= & \left(\frac{1}{2} m A I_{k-1}(t)+\frac{1}{2} m A I_{k+1}(t)-m A I_{k}(t)\right) \\
& +(1-\eta) \beta\left(\rho_{k}(t)-\left(U I_{k}(t)+A I_{k}(t)\right)\right)\left(U I_{k}(t)+A I_{k}(t)\right)+\alpha U I_{k}(t) A_{k}(t)(1-\gamma)-\gamma A I_{k}(t),
\end{aligned}
$$




\section{Wheel graphs: SIS-UA model}

For $k=1$, (Focal Point)

$$
\begin{aligned}
\frac{d A S_{1}(t)}{d t}= & \left(\sum_{i \in N, i>1} \frac{1}{3} m A S_{i}(t)-m A S_{1}(t)\right)+\alpha U S_{1}(t) A_{1}(t)\left(1-\beta\left(U I_{1}(t)+A I_{1}(t)\right)\right) \\
& -(1-\eta) \beta\left(\rho_{1}(t)-\left(U I_{1}(t)+A I_{1}(t)\right)\right)\left(U I_{1}(t)+A I_{1}(t)\right)+\gamma A I_{1}(t), \\
\frac{d A I_{1}(t)}{d t}= & \left(\sum_{i \in N, i>1} \frac{1}{3} m A I_{i}(t)-m A I_{1}(t)\right)+(1-\eta) \beta\left(\rho_{1}(t)-\left(U I_{1}(t)+A I_{1}(t)\right)\right)\left(U I_{1}(t)+A I_{1}(t)\right) \\
& +\alpha U I_{1}(t) A_{1}(t)(1-\gamma)-\gamma A I_{1}(t),
\end{aligned}
$$

For $k>1$,

$$
\begin{aligned}
\frac{d A S_{k}(t)}{d t}= & \left(\frac{1}{N-1} m A S_{1}(t)-m A S_{k}(t)+\frac{1}{3} m A S_{k+1}(t)+\frac{1}{3} m A S_{k-1}(t)\right) \\
& +\alpha U S_{k}(t) A_{k}(t)\left(1-\beta\left(U I_{k}(t)+A I_{k}(t)\right)\right)-(1-\eta) \beta\left(\rho_{k}(t)\right. \\
& \left.-\left(U I_{k}(t)+A I_{k}(t)\right)\right)\left(U I_{k}(t)+A I_{k}(t)\right)+\gamma A I_{k}(t), \\
\frac{d A I_{k}(t)}{d t}= & \left(\frac{1}{N-1} m A I_{1}(t)-m A I_{k}(t)+\frac{1}{3} m A I_{k+1}(t)+\frac{1}{3} m A I_{k-1}(t)\right) \\
& +(1-\eta) \beta\left(\rho_{k}(t)-\left(U I_{k}(t)+A I_{k}(t)\right)\right)\left(U I_{k}(t)+A I_{k}(t)\right) \\
& +\alpha U I_{k}(t) A_{k}(t)(1-\gamma)-\gamma A I_{k}(t),
\end{aligned}
$$

\section{Complete graph: SIS-UA model}

$$
\begin{aligned}
\frac{d A S_{k}(t)}{d t}= & \left(\sum_{i \in N, i \neq k} \frac{1}{N-1} m A S_{i}(t)-m A S_{k}(t)\right)+\alpha U S_{k}(t) A_{k}(t)\left(1-\beta\left(U I_{k}(t)+A I_{k}(t)\right)\right) \\
& -(1-\eta) \beta\left(\rho_{k}(t)-\left(U I_{k}(t)+A I_{k}(t)\right)\right)\left(U I_{k}(t)+A I_{k}(t)\right)+\gamma A I_{k}(t) \\
\frac{d A I_{k}(t)}{d t}= & \left(\sum_{i \in N, i \neq k} \frac{1}{N-1} m A I_{i}(t)-m A I_{k}(t)\right)+(1-\eta) \beta\left(\rho_{k}(t)-\left(U I_{k}(t)+A I_{k}(t)\right)\right)\left(U I_{k}(t)+A I_{k}(t)\right) \\
& +\alpha U I_{k}(t) A_{k}(t)(1-\gamma)-\gamma A I_{k}(t)
\end{aligned}
$$

\section{A. 2 Case 3}

\section{Star graphs: SIS-UA model}

For $k=1$, (Focal Point)

$$
\begin{aligned}
\frac{d U S_{1}(t)}{d t}= & \left(\sum_{i \in N, i>1} m U S_{i}(t)-m U S_{1}(t)\right)+(1-\eta) \beta\left(\rho_{1}(t)-\left(U I_{1}(t)+A I_{1}(t)\right)\right)\left(U I_{1}(t)+A I_{1}(t)\right)-\gamma A I_{1}(t) \\
& -\beta U S_{1}(t)\left(U I_{1}(t)+A I_{1}(t)\right)\left(1-\alpha A_{1}(t)\right)-\alpha U S_{1}(t) A_{1}(t)\left(1-\beta\left(U I_{1}(t)+A I_{1}(t)\right)\right)+\gamma U I_{1}(t),
\end{aligned}
$$




$$
\begin{gathered}
\frac{d A S_{1}(t)}{d t}=\left(\sum_{i \in N, i>1} m A S_{i}(t)-m A S_{1}(t)\right)+\alpha U S_{1}(t) A_{1}(t)\left(1-\beta\left(U I_{1}(t)+A I_{1}(t)\right)\right) \\
-(1-\eta) \beta\left(\rho_{1}(t)-\left(U I_{1}(t)+A I_{1}(t)\right)\right)\left(U I_{1}(t)+A I_{1}(t)\right)+\gamma A I_{1}(t) \\
\frac{d U I_{1}(t)}{d t}=\left(\sum_{i \in N, i>1} m U I_{i}(t)-m U I_{1}(t)\right)+\beta U S_{1}(t)\left(U I_{1}(t)+A I_{1}(t)\right)\left(1-\alpha A_{1}(t)\right)-\gamma U I_{1}(t) \\
\frac{d A I_{1}(t)}{d t}=\left(\sum_{i \in N, i>1} m A I_{i}(t)-m A I_{1}(t)\right)+(1-\eta) \beta\left(\rho_{1}(t)-\left(U I_{1}(t)+A I_{1}(t)\right)\right)\left(U I_{1}(t)+A I_{1}(t)\right)-\gamma A I_{1}(t),
\end{gathered}
$$

For $k>1$,

$$
\begin{gathered}
\begin{aligned}
& \frac{d U S_{k}(t)}{d t}=\left(\frac{1}{N-1} m U S_{1}(t)-m U S_{k}(t)\right)-\beta U S_{k}(t)\left(U I_{k}(t)+A I_{k}(t)\right)\left(1-\alpha A_{k}(t)\right) \\
&-\alpha U S_{k}(t) A_{k}(t)\left(1-\beta\left(U I_{k}(t)+A I_{k}(t)\right)\right)+\gamma U I_{k}(t) \\
& \frac{d A S_{k}(t)}{d t}=\left(\frac{1}{N-1} m A S_{1}(t)-m A S_{k}(t)\right)+\alpha U S_{k}(t) A_{k}(t)\left(1-\beta\left(U I_{k}(t)+A I_{k}(t)\right)\right) \\
&-(1-\eta) \beta\left(\rho_{k}(t)-\left(U I_{k}(t)+A I_{k}(t)\right)\right)\left(U I_{k}(t)+A I_{k}(t)\right)+\gamma A I_{k}(t) \\
& \frac{d U I_{k}(t)}{d t}=\left(\frac{1}{N-1} m U I_{1}(t)-m U I_{k}(t)\right)+\beta U S_{k}(t)\left(U I_{k}(t)+A I_{k}(t)\right)\left(1-\alpha A_{k}(t)\right)-\gamma U I_{k}(t) \\
& \frac{d A I_{k}(t)}{d t}=\left(\frac{1}{N-1} m A I_{1}(t)-m A I_{k}(t)\right)+(1-\eta) \beta\left(\rho_{k}(t)-\left(U I_{k}(t)+A I_{k}(t)\right)\right)\left(U I_{k}(t)+A I_{k}(t)\right)-\gamma A I_{k}(t)
\end{aligned}
\end{gathered}
$$

\section{Cycle graph: SIS-UA model}

$$
\begin{gathered}
\begin{aligned}
\frac{d U S_{k}(t)}{d t}= & \left(\frac{1}{2} m U S_{k-1}(t)+\frac{1}{2} m U S_{k+1}(t)-m U S_{k}(t)\right)-\beta U S_{k}(t)\left(U I_{k}(t)+A I_{k}(t)\right)\left(1-\alpha A_{k}(t)\right) \\
& -\alpha U S_{k}(t) A_{k}(t)\left(1-\beta\left(U I_{k}(t)+A I_{k}(t)\right)\right)+\gamma U I_{k}(t) \\
\frac{d A S_{k}(t)}{d t}= & \left(\frac{1}{2} m A S_{k-1}(t)+\frac{1}{2} m A S_{k+1}(t)-m A S_{k}(t)\right)+\alpha U S_{k}(t) A_{k}(t)\left(1-\beta\left(U I_{k}(t)+A I_{k}(t)\right)\right) \\
& -(1-\eta) \beta\left(\rho_{k}(t)-\left(U I_{k}(t)+A I_{k}(t)\right)\right)\left(U I_{k}(t)+A I_{k}(t)\right)+\gamma A I_{k}(t) \\
\frac{d U I_{k}(t)}{d t}=\left(\begin{array}{c}
\left.\frac{1}{2} m U I_{k-1}(t)+\frac{1}{2} m U I_{k+1}(t)-m U I_{k}(t)\right)+\beta U S_{k}(t)\left(U I_{k}(t)+A I_{k}(t)\right)\left(1-\alpha A_{k}(t)\right)-\gamma U I_{k}(t) \\
\frac{d A I_{k}(t)}{d t}=
\end{array}\right. & \left(\frac{1}{2} m A I_{k-1}(t)+\frac{1}{2} m A I_{k+1}(t)-m A I_{k}(t)\right)+(1-\eta) \beta\left(\rho_{k}(t)\left(U I_{k}(t)+A I_{k}(t)\right)\right) \\
& \times\left(U I_{k}(t)+A I_{k}(t)\right)-\gamma A I_{k}(t)
\end{aligned}
\end{gathered}
$$




\section{Wheel graphs: SIS-UA model}

For $k=1$, (Focal Point)

$$
\begin{gathered}
\frac{d U S_{1}(t)}{d t}=\left(\sum_{i \in N, i>1} \frac{1}{3} m U S_{i}(t)-m U S_{1}(t)\right)-\beta U S_{1}(t)\left(U I_{1}(t)+A I_{1}(t)\right)\left(1-\alpha A_{1}(t)\right) \\
-\alpha U S_{1}(t) A_{1}(t)\left(1-\beta\left(U I_{1}(t)+A I_{1}(t)\right)\right)+\gamma U I_{1}(t), \\
\begin{aligned}
& \frac{d A S_{1}(t)}{d t}=\left(\sum_{i \in N, i>1} \frac{1}{3} m A S_{i}(t)-m A S_{1}(t)\right)+\alpha U S_{1}(t) A_{1}(t)\left(1-\beta\left(U I_{1}(t)+A I_{1}(t)\right)\right) \\
&-(1-\eta) \beta\left(\rho_{1}(t)-\left(U I_{1}(t)+A I_{1}(t)\right)\right)\left(U I_{1}(t)+A I_{1}(t)\right)+\gamma A I_{1}(t), \\
& \frac{d U I_{1}(t)}{d t}=\left(\sum_{i \in N, i>1} \frac{1}{3} m A S_{i}(t)-m A S_{1}(t)\right)+\beta U S_{1}(t)\left(U I_{1}(t)+A I_{1}(t)\right)\left(1-\alpha A_{1}(t)\right)-\gamma U I_{1}(t),(\mathrm{A} .27) \\
& \frac{d A I_{1}(t)}{d t}=\left(\sum_{i \in N, i>1} \frac{1}{3} m A I_{i}(t)-m A I_{1}(t)\right)+(1-\eta) \beta\left(\rho_{1}(t)-\left(U I_{1}(t)+A I_{1}(t)\right)\right)\left(U I_{1}(t)+A I_{1}(t)\right)-\gamma A I_{1}(t),
\end{aligned}
\end{gathered}
$$

For $k>1$,

$$
\begin{aligned}
\frac{d U S_{k}(t)}{d t}= & \left(\frac{1}{N-1} m U S_{1}(t)-m U S_{k}(t)+\frac{1}{3} m U S_{k+1}(t)+\frac{1}{3} m U S_{k-1}(t)\right) \\
& -\beta U S_{k}(t)\left(U I_{k}(t)+A I_{k}(t)\right)\left(1-\alpha A_{k}(t)\right)-\alpha U S_{k}(t) A_{k}(t)\left(1-\beta\left(U I_{k}(t)+A I_{k}(t)\right)\right)+\gamma U I_{k}(t)
\end{aligned}
$$

$$
\begin{aligned}
\frac{d A S_{k}(t)}{d t}= & \left(\frac{1}{N-1} m A S_{1}(t)-m A S_{k}(t)+\frac{1}{3} m A S_{k+1}(t)+\frac{1}{3} m A S_{k-1}(t)\right) \\
& +\alpha U S_{k}(t) A_{k}(t)\left(1-\beta\left(U I_{k}(t)+A I_{k}(t)\right)\right)-(1-\eta) \beta A S_{k}(t)\left(U I_{k}(t)+A I_{k}(t)\right)+\gamma A I_{k}(t),
\end{aligned}
$$

$$
\begin{aligned}
\frac{d U I_{k}(t)}{d t}= & \left(\frac{1}{N-1} m U I_{1}(t)-m U I_{k}(t)+\frac{1}{3} m U I_{k+1}(t)+\frac{1}{3} m U I_{k-1}(t)\right) \\
& +\beta U S_{k}(t)\left(U I_{k}(t)+A I_{k}(t)\right)\left(1-\alpha A_{k}(t)\right)-\gamma U I_{k}(t), \\
\frac{d A I_{k}(t)}{d t}= & \left(\frac{1}{N-1} m A I_{1}(t)-m A I_{k}(t)+\frac{1}{3} m A I_{k+1}(t)+\frac{1}{3} m A I_{k-1}(t)\right) \\
+ & (1-\eta) \beta\left(\rho_{k}(t)-\left(U I_{k}(t)+A I_{k}(t)\right)\right)\left(U I_{k}(t)+A I_{k}(t)\right)-\gamma A I_{k}(t),
\end{aligned}
$$

\section{Complete graph: SIS-UA model}

$$
\begin{aligned}
\frac{d U S_{k}(t)}{d t}= & \left(\sum_{i \in N, i \neq k} \frac{1}{N-1} m U S_{i}(t)-m U S_{k}(t)\right)-\beta U S_{k}(t)\left(U I_{k}(t)+A I_{k}(t)\right)\left(1-\alpha A_{k}(t)\right) \\
& -\alpha U S_{k}(t) A_{k}(t)\left(1-\beta\left(U I_{k}(t)+A I_{k}(t)\right)\right)+\gamma U I_{k}(t),
\end{aligned}
$$




$$
\begin{aligned}
& \frac{d A S_{k}(t)}{d t}=\left(\sum_{i \in N, i \neq k} \frac{1}{N-1} m A S_{i}(t)-m A S_{k}(t)\right)+\alpha U S_{k}(t) A_{k}(t)\left(1-\beta\left(U I_{k}(t)+A I_{k}(t)\right)\right) \\
&-(1-\eta) \beta A S_{k}(t)\left(U I_{k}(t)+A I_{k}(t)\right)+\gamma A I_{k}(t), \\
& \frac{d U I_{k}(t)}{d t}=\left(\sum_{i ? N, i ? k} \frac{1}{N-1} m U I_{i}(t)-m U I_{k}(t)\right)+\beta U S_{k}(t)\left(U I_{k}(t)+A I_{k}(t)\right)\left(1-\alpha A_{k}(t)\right)-\gamma U I_{k}(t), \\
& \frac{d A I_{k}(t)}{d t}=\left(\sum_{i \in N, i \neq k} \frac{1}{N-1} m A I_{i}(t)-m A I_{k}(t)\right)+(1-\eta) \beta\left(\rho_{k}(t)-\left(U I_{k}(t)+A I_{k}(t)\right)\right) \\
& \\
& \times\left(U I_{k}(t)+A I_{k}(t)\right)-\gamma A I_{k}(t),
\end{aligned}
$$

\section{A.3 Case 5}

\section{Star graphs: SIS-UA model}

For $k=1$, (Focal Point)

$$
\begin{aligned}
\frac{d A S_{1}(t)}{d t}= & \left(\sum_{i \in N, i>1} m A S_{i}(t)-m A S_{1}(t)\right)+\alpha U S_{1}(t) A_{1}(t)\left(1-\beta\left(U I_{1}(t)+A I_{1}(t)\right)\right) \\
& -(1-\eta) \beta\left(\rho_{1}(t)-\left(U I_{1}(t)+A I_{1}(t)\right)\right)+\gamma A I_{1}(t),
\end{aligned}
$$

For $k>1$,

$$
\begin{aligned}
\frac{d A S_{k}(t)}{d t}= & \left(\frac{1}{N-1} m A S_{1}(t)-m A S_{k}(t)\right)+\alpha U S_{k}(t) A_{k}(t)\left(1-\beta\left(U I_{k}(t)+A I_{k}(t)\right)\right) \\
& -(1-\eta) \beta\left(\rho_{k}(t)-\left(U I_{k}(t)+A I_{k}(t)\right)\right)\left(U I_{k}(t)+A I_{k}(t)\right)+\gamma A I_{k}(t),
\end{aligned}
$$

\section{Cycle graph: SIS-UA model}

$$
\begin{aligned}
\frac{d A S_{k}(t)}{d t}= & \left(\frac{1}{2} m A S_{k-1}(t)+\frac{1}{2} m A S_{k+1}(t)-m A S_{k}(t)\right)+\alpha U S_{k}(t) A_{k}(t)\left(1-\beta\left(U I_{k}(t)+A I_{k}(t)\right)\right) \\
& -(1-\eta) \beta\left(\rho_{k}(t)-\left(U I_{k}(t)+A I_{k}(t)\right)\right)\left(U I_{k}(t)+A I_{k}(t)\right)+\gamma A I_{k}(t),
\end{aligned}
$$

\section{Wheel graphs: SIS-UA model}

For $k=1$, (Focal Point)

$$
\begin{aligned}
\frac{d A S_{1}(t)}{d t}= & \left(\sum_{i \in N, i>1} \frac{1}{3} m A S_{i}(t)-m A S_{1}(t)\right)+\alpha U S_{1}(t) A_{1}(t)\left(1-\beta\left(U I_{1}(t)+A I_{1}(t)\right)\right) \\
& -(1-\eta) \beta\left(\rho_{1}(t)-\left(U I_{1}(t)+A I_{1}(t)\right)\right)\left(U I_{1}(t)+A I_{1}(t)\right)+\gamma A I_{1}(t),
\end{aligned}
$$

For $k>1$,

$$
\begin{aligned}
\frac{d A S_{k}(t)}{d t}= & \left(\frac{1}{N-1} m A S_{1}(t)-m A S_{k}(t)+\frac{1}{3} m A S_{k+1}(t)+\frac{1}{3} m A S_{k-1}(t)\right)+\alpha U S_{k}(t) A_{k}(t) \\
& \times\left(1-\beta\left(U I_{k}(t)+A I_{k}(t)\right)\right)-(1-\eta) \beta\left(\rho_{k}(t)-\left(U I_{k}(t)+A I_{k}(t)\right)\right)\left(U I_{k}(t)+A I_{k}(t)\right)+\gamma A I_{k}(t)
\end{aligned}
$$

\section{Complete graph: SIS-UA model}

$$
\begin{aligned}
\frac{d A S_{k}(t)}{d t}= & \left(\sum_{i \in N, i \neq k} \frac{1}{N-1} m A S_{i}(t)-m A S_{k}(t)\right)+\alpha U S_{k}(t) A_{k}(t)\left(1-\beta\left(U I_{k}(t)+A I_{k}(t)\right)\right) \\
& -(1-\eta) \beta\left(\rho_{k}(t)-\left(U I_{k}(t)+A I_{k}(t)\right)\right)\left(U I_{k}(t)+A I_{k}(t)\right)+\gamma A I_{k}(t),
\end{aligned}
$$




\section{A.4 Case 6}

\section{Star graphs: SIS-UA model}

For $k=1$, (Focal Point)

$$
\frac{d U I_{1}(t)}{d t}=\left(\sum_{i \in N, i>1} m U I_{i}(t)-m U I_{1}(t)\right)+\beta U S_{k}(t)\left(U I_{1}(t)+A I_{1}(t)\right)\left(1-\alpha A_{1}(t)\right)-\gamma U I_{1}(t),
$$

For $k>1$,

$$
\frac{d U I_{k}(t)}{d t}=\left(\frac{1}{N-1} m U I_{1}(t)-m U I_{k}(t)\right)+\beta U S_{k}(t)\left(U I_{k}(t)+A I_{k}(t)\right)\left(1-\alpha A_{k}(t)\right)-\gamma U I_{k}(t),
$$

\section{Cycle graph: SIS-UA model}

$$
\frac{d U I_{k}(t)}{d t}=\left(\frac{1}{2} m U I_{k-1}(t)+\frac{1}{2} m U I_{k+1}(t)-m U I_{k}(t)\right)+\beta U S_{k}(t)\left(U I_{k}(t)+A I_{k}(t)\right)\left(1-\alpha A_{k}(t)\right)-\gamma U I_{k}(t)
$$

\section{Wheel graphs: SIS-UA model}

For $k=1$, (Focal Point)

$$
\frac{d U I_{1}(t)}{d t}=\left(\sum_{i \in N, i>1} \frac{1}{3} m A S_{i}(t)-m A S_{1}(t)\right)+\beta U S_{k}(t)\left(U I_{1}(t)+A I_{1}(t)\right)\left(1-\alpha A_{1}(t)\right)-\gamma U I_{1}(t),
$$

For $k>1$,

$$
\begin{aligned}
\frac{d U I_{k}(t)}{d t}= & \left(\frac{1}{N-1} m U I_{1}(t)-m U I_{k}(t)+\frac{1}{3} m U I_{k+1}(t)+\frac{1}{3} m U I_{k-1}(t)\right)+\beta U S_{k}(t) \\
& \times\left(U I_{k}(t)+A I_{k}(t)\right)\left(1-\alpha A_{k}(t)\right)-\gamma U I_{k}(t),
\end{aligned}
$$

\section{Complete graph: SIS-UA model}

$$
\frac{d U I_{k}(t)}{d t}=\left(\sum_{i \in N, i \neq k} \frac{1}{N-1} m U I_{i}(t)-m U I_{k}(t)\right)+\beta U S_{k}(t)\left(U I_{k}(t)+A I_{k}(t)\right)\left(1-\alpha A_{k}(t)\right)-\gamma U I_{k}(t),
$$

